Submitted to the Annals of Applied Statistics

arXiv: arXiv: 0000.0000

\title{
SUPPLEMENTAL MATERIAL: FORECASTING SEASONAL INFLUENZA WITH A STATE-SPACE SIR MODEL
}

\author{
By Dave Osthus ${ }^{*} \dagger$, Kyle S. Hickmann*, Petruţa C. Caragea ${ }^{\dagger}$, \\ Dave Higdon ${ }^{\ddagger}, *$, And Sara Y. Del Valle* \\ Los Alamos National Laboratory*, Iowa State University ${ }^{\dagger}$, and Virginia \\ Tech $^{\ddagger}$
}

1. Fourth order Runge-Kutta approximation details. When the explicit solution to Equation 4.2 of the main text is not known, a numerical approximation to $f\left(\theta_{t-1}, \beta, \gamma\right)$ must be specified. We chose to define $f\left(\theta_{t-1}, \beta, \gamma\right)$ as the fourth order Runge-Kutta (RK4) approximation to Equation 4.2. The details of $f\left(\theta_{t-1}, \beta, \gamma\right)$ are as follows:

$$
f\left(\theta_{t-1}, \beta, \gamma\right)=\left(\begin{array}{c}
\theta_{t-1}^{S}+\frac{1}{6}\left[k_{t-1}^{S 1}+2 k_{t-1}^{S 2}+2 k_{t-1}^{S 3}+k_{t-1}^{S 4}\right] \\
\theta_{t-1}^{I}+\frac{1}{6}\left[k_{t-1}^{I 1}+2 k_{t-1}^{I 2}+2 k_{t-1}^{I 3}+k_{t-1}^{I 4}\right] \\
\theta_{t-1}^{R}+\frac{1}{6}\left[k_{t-1}^{R 1}+2 k_{t-1}^{R 2}+2 k_{t-1}^{R 3}+k_{t-1}^{R 4}\right]
\end{array}\right),
$$

where

$$
\begin{aligned}
k_{t}^{S 1} & =-\beta \theta_{t}^{S} \theta_{t}^{I} \\
k_{t}^{S 2} & =-\beta\left[\theta_{t}^{S}+.5 k_{t}^{S 1}\right]\left[\theta_{t}^{I}+.5 k_{t}^{I 1}\right] \\
k_{t}^{S 3} & =-\beta\left[\theta_{t}^{S}+.5 k_{t}^{S 2}\right]\left[\theta_{t}^{I}+.5 k_{t}^{I 2}\right] \\
k_{t}^{S 4} & =-\beta\left[\theta_{t}^{S}+k_{t}^{S 3}\right]\left[\theta_{t}^{I}+k_{t}^{I 3}\right], \\
k_{t}^{I 1} & =\beta \theta_{t}^{S} \theta_{t}^{I}-\gamma \theta_{t}^{I} \\
k_{t}^{I 2} & =\beta\left[\theta_{t}^{S}+.5 k_{t}^{S 1}\right]\left[\theta_{t}^{I}+.5 k_{t}^{I 1}\right]-\gamma\left[\theta_{t}^{I}+.5 k_{t}^{I 1}\right] \\
k_{t}^{I 3} & =\beta\left[\theta_{t}^{S}+.5 k_{t}^{S 2}\right]\left[\theta_{t}^{I}+.5 k_{t}^{I 2}\right]-\gamma\left[\theta_{t}^{I}+.5 k_{t}^{I 2}\right] \\
k_{t}^{I 4} & =\beta\left[\theta_{t}^{S}+k_{t}^{S 3}\right]\left[\theta_{t}^{I}+k_{t}^{I 3}\right]-\gamma\left[\theta_{t}^{I}+k_{t}^{I 3}\right], \\
k_{t}^{R 1} & =\gamma \theta_{t}^{I} \\
k_{t}^{R 2} & =\gamma\left[\theta_{t}^{I}+.5 k_{t}^{I 1}\right] \\
k_{t}^{R 3} & =\gamma\left[\theta_{t}^{I}+.5 k_{t}^{I 2}\right] \\
k_{t}^{R 4} & =\gamma\left[\theta_{t}^{I}+k_{t}^{I 3}\right] .
\end{aligned}
$$


2. Regression parameter estimates. As described in Section 6.5 of the main text, a subset of a fourth-degree polynomial regression of $\log (\beta)$ on $\log (P T), \log \left(I_{0}\right)$, and $\log (\rho)$ was fit to estimate the relationship between $\mathrm{PT}$ and $\phi$. The regression details are shown in Table 1 .

TABLE 1

\begin{tabular}{rrrr} 
Polynomial regression of $\log (\beta)$ on $\log (P T), \log \left(I_{0}\right)$, and $\log (\rho)$ \\
\hline Covariate & Parameter & Estimate & Std. Error \\
\hline $\operatorname{Intercept}$ & $\tau_{1}$ & -49.7540 & 1.4653 \\
$\log (P T)$ & $\tau_{2}$ & -0.9577 & 0.0072 \\
$\log (P T)^{2}$ & $\tau_{3}$ & -0.0065 & 0.0013 \\
$\log \left(I_{0}\right)$ & $\tau_{4}$ & -9.4896 & 0.3327 \\
$\log \left(I_{0}\right)^{2}$ & $\tau_{5}$ & -0.3761 & 0.0184 \\
$\log (\rho)$ & $\tau_{6}$ & -590.0001 & 22.2718 \\
$\log (\rho)^{2}$ & $\tau_{7}$ & -2537.6102 & 117.7408 \\
$\log (\rho)^{3}$ & $\tau_{8}$ & -4756.1828 & 259.2917 \\
$\log (\rho)^{4}$ & $\tau_{9}$ & -3265.2458 & 202.6393 \\
\hline $\log \left(I_{0}\right) * \log (\rho)$ & $\tau_{10}$ & -102.2665 & 5.0522 \\
$\log \left(I_{0}\right)^{2} * \log (\rho)$ & $\tau_{11}$ & -4.0162 & 0.2793 \\
$\log \left(I_{0}\right) * \log (\rho)^{2}$ & $\tau_{12}$ & -430.9596 & 26.7017 \\
$\log \left(I_{0}\right)^{2} * \log (\rho)^{2}$ & $\tau_{13}$ & -16.7104 & 1.4755 \\
$\log \left(I_{0}\right) * \log (\rho)^{3}$ & $\tau_{14}$ & -798.3443 & 58.7927 \\
$\log \left(I_{0}\right)^{2} * \log (\rho)^{3}$ & $\tau_{15}$ & -30.6638 & 3.2478 \\
$\log \left(I_{0}\right) * \log (\rho)^{4}$ & $\tau_{16}$ & -543.8857 & 45.9414 \\
$\log \left(I_{0}\right)^{2} * \log (\rho)^{4}$ & $\tau_{17}$ & -20.7459 & 2.5373 \\
\hline
\end{tabular}

3. Sampling from the prior predictive density. The prescription to sample from the prior predictive density of Equation 6.1 of the main text, $\left[y_{1: T}\right]$, is outlined in Algorithm 1. 


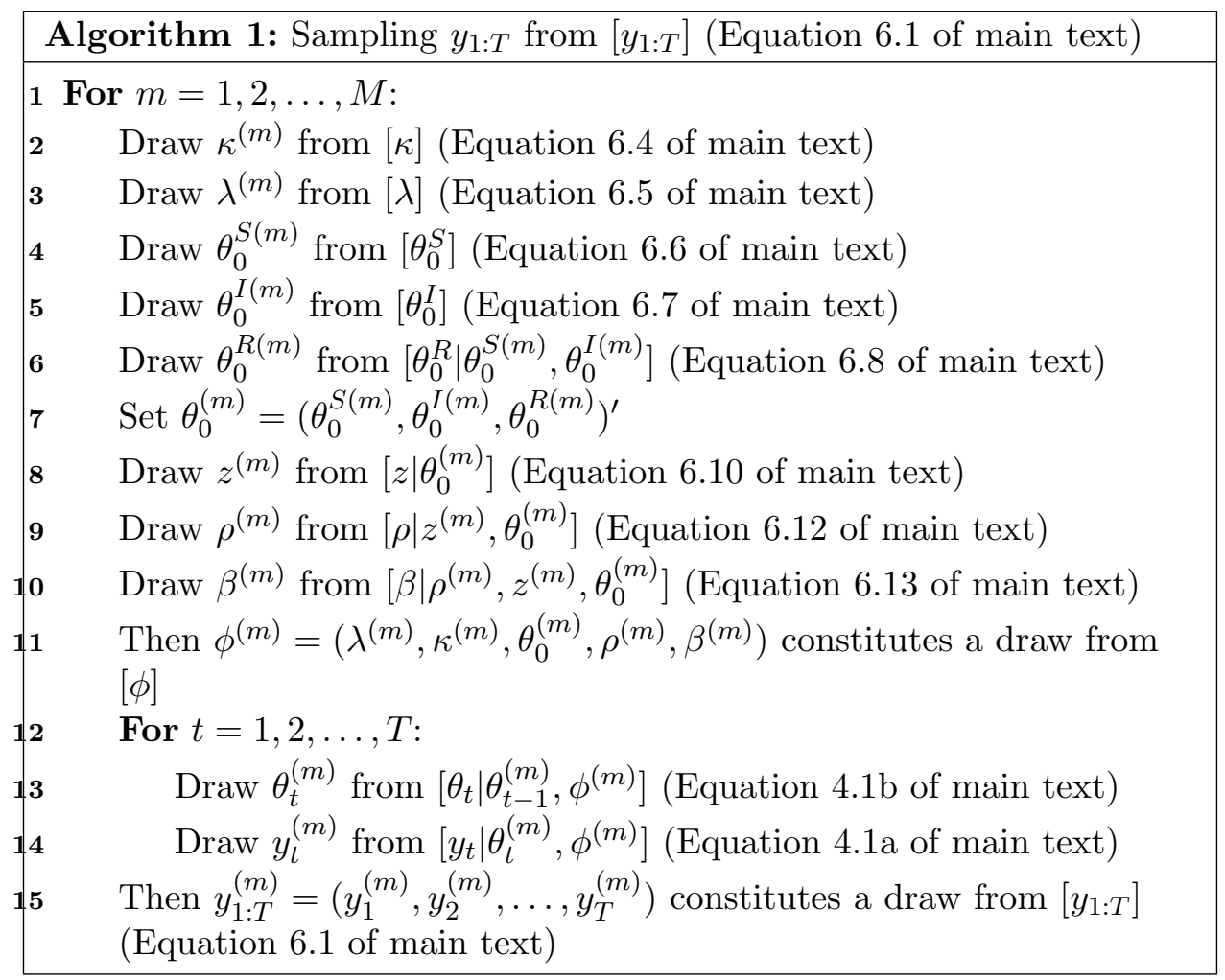

4. Sampling from the posterior predictive density. Sampling from the posterior predictive density of Equation 5.2 of the main text, $\left[y_{t^{\prime}+1: T} \mid y_{1: t^{\prime}}\right]$, is outlined in Algorithm 2.

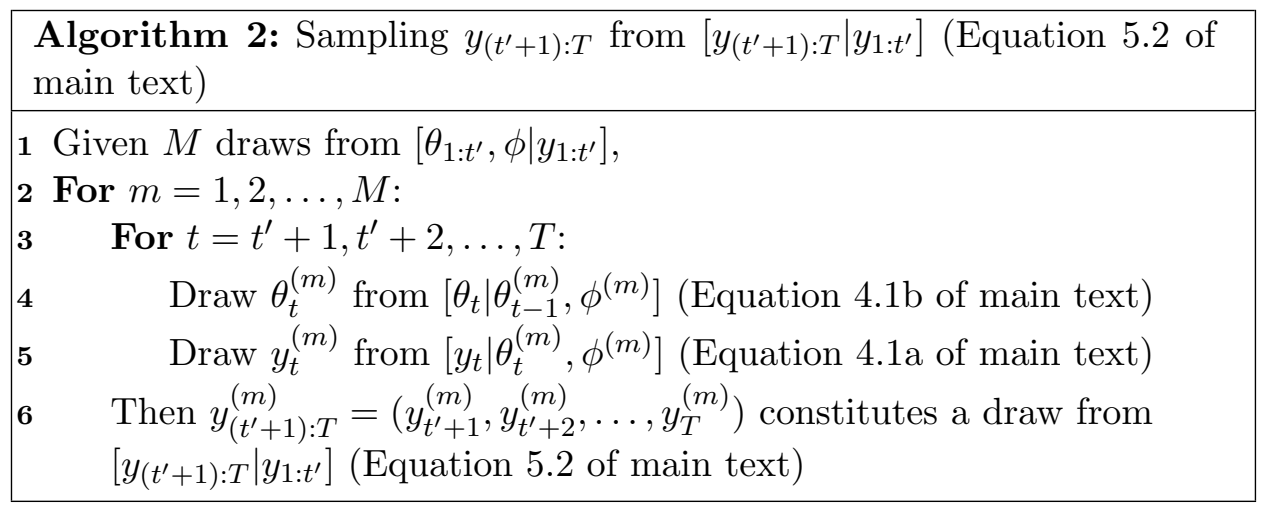


5. MCMC diagnostics. Gibbs sampling can have difficulties sampling from compartmental models due to highly correlated posterior structures. The DBSSM is not a compartmental model, but shares some of these difficulties. In this application, Gibbs sampling proved tractable in part, because of the parameter transformation $\rho=\gamma / \beta$. Parameters $\rho$ and $\beta$ are less correlated than $\gamma$ and $\beta$. This is illustrated in Figure 1.
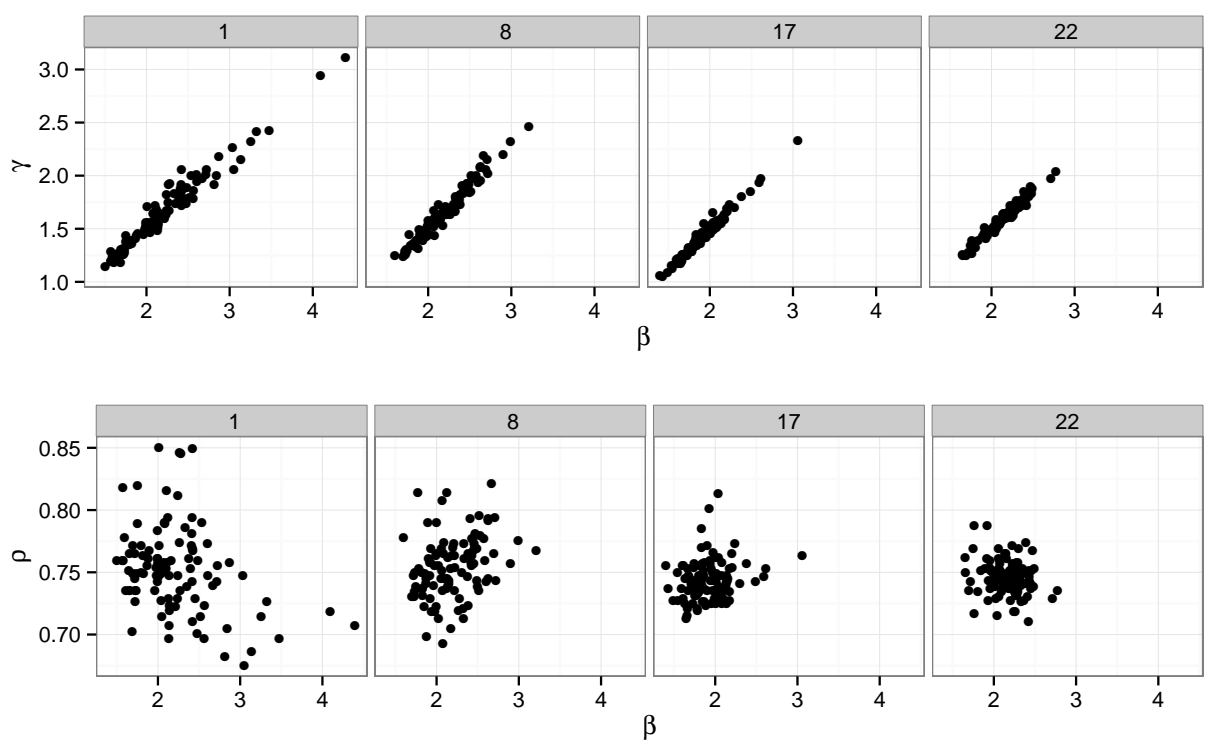

FIG 1. A sample of 100 posterior draws of $\gamma$ and $\beta$ (top) and $\rho$ and $\beta$ (bottom) corresponding to the 2010 nationwide, ILI+ posterior distributions for $t^{\prime}=1,8$, 17, and 22.

For the posterior distributions corresponding to $t^{\prime}=1,8,17$, and 22 of 2010 nationwide ILI+, we assess chain mixing and stationarity for various estimands of interest following the recommendations of Sections 11.4 and 11.5 of Gelman et al. (2014). Specifically, after burn-in and thinning, we split each of the four chains in half, resulting in eight chains of length 2500 . We calculate the Gelman-Rubin diagnostic, $\hat{R}$, for these eight chains, checking that $\hat{R}$ is less than 1.1 for all scalar estimands of interest (Gelman and Rubin, 1992). Furthermore, we calculate the effective sample size (ESS) for the concatenated four chains. Table 2 presents $\hat{R}$ and ESS corresponding to the 2010 nationwide, ILI+ posterior distributions for $t^{\prime}=1,8,17$, and 22 . 
TABle 2

Gelman-Rubin diagnostic $(\hat{R})$ and effective sample size (ESS) for the four posterior distributions corresponding to the 2010 nationwide, ILI+ posterior distributions for $t^{\prime}=$ 1, 8, 17, and 22.

\begin{tabular}{rr|ccccccccc}
\hline $\boldsymbol{t}^{\prime}$ & & $\boldsymbol{\beta}$ & $\boldsymbol{\gamma}$ & $\boldsymbol{\rho}$ & $\boldsymbol{\kappa}$ & $\boldsymbol{\lambda}$ & $\boldsymbol{\theta}_{\mathbf{1}}^{\boldsymbol{I}}$ & $\boldsymbol{\theta}_{\mathbf{8}}^{\boldsymbol{I}}$ & $\boldsymbol{\theta}_{\mathbf{1 7}}^{\boldsymbol{I}}$ & $\boldsymbol{\theta}_{\mathbf{2 2}}^{\boldsymbol{I}}$ \\
\hline \multirow{2}{*}{1} & $\hat{R}$ & 1.00 & 1.00 & 1.00 & 1.00 & 1.00 & 1.03 & & & \\
& $\mathrm{ESS}$ & 11068 & 10063 & 17861 & 18483 & 7923 & 804 & & & \\
\hline \multirow{2}{*}{8} & $\hat{R}$ & 1.00 & 1.00 & 1.00 & 1.00 & 1.00 & 1.01 & 1.01 & & \\
& $\mathrm{ESS}$ & 4525 & 5537 & 13914 & 3661 & 4298 & 589 & 2858 & & \\
\hline \multirow{2}{*}{17} & $\hat{R}$ & 1.03 & 1.03 & 1.00 & 1.01 & 1.01 & 1.02 & 1.01 & 1.01 & \\
& $\mathrm{ESS}$ & 340 & 398 & 4860 & 624 & 1019 & 685 & 1581 & 1956 & \\
\hline \multirow{2}{*}{22} & $\hat{R}$ & 1.01 & 1.01 & 1.01 & 1.04 & 1.01 & 1.01 & 1.00 & 1.00 & 1.01 \\
& $\mathrm{ESS}$ & 554 & 752 & 502 & 625 & 1136 & 734 & 2330 & 3051 & 1262 \\
\hline
\end{tabular}

\section{References.}

Gelman, A., Rubin, D. B. (1992). Inference from iterative simulation using multiple sequences. Statistical science, (7):457-472.

Gelman, A., Carlin, J. B., Stern, H. S., Dunson, D. B., Vehtari, A., and Rubin, D. B. (2014). Bayesian data analysis - Third edition. Boca Raton, FL, USA: Chapman \& Hall/CRC. pages 281-288. 